\title{
Advanced evaluation of simultaneous train formation methods based on fuzzy compromise programing
}

\author{
Ivan Belošević ${ }^{1, *}$, Sanjin Milinković ${ }^{1}$, Miloš Ivić $^{1}$, and Peter Marton ${ }^{2}$ \\ ${ }^{1}$ University of Belgrade, Vojvode Stepe, 305, 11000, Belgrade, Serbia \\ ${ }^{2}$ University of Žilina, Univerzitná, 1, 01026, Žilina, Slovakia
}

\begin{abstract}
Multi-group trains have potential to take a substantial segment in realizing wagonload services. Multi-group train formation is a complex marshalling procedure which composes a train by sorting wagons according to their destinations. The order of wagons corresponds to the disposition of destinations on the train route. The determination of rational sorting schedules should be based on a comprehensive approach addressing different quantitative and qualitative indicators which are conflicting and uncertain. In order to address these conditions, we proposed a new approach for fuzzy multi-criteria evaluation of simultaneous train formation methods. In this paper we evaluate simultaneous train formation methods and provide recommendations for the rational application of elementary, triangular and geometric sorting schedules regarding different scales of sorting task complexity.
\end{abstract}

\section{Introduction}

In the context of reducing negative impacts from transport sector, railways are commonly promoted as an efficient and sustainable transport alternative. Moreover, the modal shift to railways is embedded in transportation policies at different national and EU levels [1,2]. In order to be more competitive to road transport, infrastructural and operational management of marshalling yards and terminals need to be improved $[3,4]$.

Multi-group train formation is a complex marshalling procedure which composes a train by sorting wagons according to their destinations. This sorting gives the possibility of adjusting wagonload services to customers' needs. Unfortunately, the sorting has its disadvantages: it demands additional sidings and increases wagon layover which results in the increase of transport costs and the risk of goods damaging.

Currently, multi-group trains play a significant role in the industrial railway transport, which is a link in the transport chain between the industry and public transport. Industrial railways are characterized by a highly developed track network, with a number of manipulation points. In industrial trains, wagons are grouped according to the order of

\footnotetext{
* Corresponding author: i.belosevic@sf.bg.ac.rs
} 
manipulation points, to enable faster and simpler delivery. Multi-group trains are used in local transport serving a line section between two marshalling yards in order to deliver and collect wagons from intermediate stations. Finally, multi-group trains could be also used in long-distance transport connecting a number of terminals in a region in order to consolidate small flows of wagons along the transport routes.

Methods for multi-group train formation are basically divided into methods for consecutive and simultaneous train formation such as presented in [6]. Methods for consecutive formation are often used in railways and offer train formation one after another. They are also known as sorting by train methods. In these methods, the wagons for a common train are initially accumulated at the same track. After finishing the accumulation for each train individually, wagons are sorted at special sorting sidings according to the belonging groups. The duration of the corresponding train formation procedure directly depends on the number of outbound trains. Within simultaneous train formation methods, wagons are sorted according to the blocks that encompass same groups in all outbound trains. This alternation makes the sorting procedure insensitive to the number of trains. Simultaneous methods with fixed sorting schedules are known as elementary, triangular and geometric methods and their performances are already examined using simulation modelling in [7]. The optimization models for minimizing shunting volume, either expressed as a number of movements or pull-out operations, are developed in e.g. $[8,9]$.

However, the determination of rational sorting schedules should not be based only on shunting volume. It should be rather based on more comprehencive approach considering wide range of technical and technological aspects including additional performance indicators such as required number of tracks, duration of processing time and complexity of shunting operations. These additional performances could be expresed using quantitative and qualitative indicators which commonly include a factor of uncertainty in assessing their values.

In order to address these conflicting and uncertain conditions, we propose a new fuzzy multi-criteria decision-making approach for evaluating sorting schedules. The proposed approach is based on the fuzzy VIKOR method and is used to evaluate aforementioned simultaneous methods. VIKOR is well-known multi-criteria decision method based on compromise programming, while fuzzy numbers are used to mathematically define the uncertainties. VIKOR in fuzzy environment was applied for solving various problems (see e.g. [10]) including railway problems such as presented in [11 -13].

The paper is organized as follows. After the introductions in Chapter 1, a brief overview of the VIKOR method and the theory of fuzzy sets is given in Chapter 2. Chapter 3 presents the proposed fuzzy multi-criteria approach to evaluate sorting schedules, while the applicability of the proposed approach is presented in Chapter 4. The final chapter presents conclusions.

\section{Preliminaries}

The proposed approach to evaluate simultaneous sorting schedules is based on the application of VIKOR methods in a fuzzy environment. In this regard, this chapter gives a brief overview of the VIKOR method and the theory of fuzzy sets.

\subsection{VIKOR method}

The VIKOR method was developed as a compromise programming method for resolving discrete problems with conflict and diverse criteria. The theoretical background based on 
the comparison with the several other outstanding multi-criteria decision making methods was presented in [14].

The VIKOR method ranks alternatives and determines the compromise solution as the closest alternative to an ideal point using a measure:

$$
L_{j}^{p}=\left\{\Sigma_{i=1}^{n}\left[\frac{w_{i}\left[f_{i}^{*}-f_{i j}\right.}{f_{i}^{*}-f_{i}^{2}}\right]^{p}\right\}^{1 / p} 1 \leq p \leq \infty
$$

where:

$f_{i j}$ - the value of $t$ - criteria function for $j$-variant solution, $i=1, \ldots, n$ i $j=1, \ldots, m$;

$f_{i}^{*}$ and $f_{i}^{-}-$the best and the worst value of the criteria function;

$w_{i}$-weight coefficients of selected criteria, $w_{i} \geq 0$.

In the VIKOR method, $L_{j}^{1}$ and $L_{j}^{\infty}$ are used to formulate ranking measures. The solution obtained by $\min L_{j}^{1}$ is with a maximum group utility ("majority" rule), and the solution obtained by $\min L_{j}^{\infty}$ is with a minimum individual regret of the "opponent". The compromise ranking measure aggregates $L_{j}^{1}$ and $L_{j}^{\infty}$ with weight $\vartheta$, providing a balance between a maximum group utility of the majority and a minimum of individual regret of the opponent. The main result is the ranking list of alternatives obtained by the compromise ranking measure.

\subsection{Fuzzy Sets Theory}

The theory of fuzzy sets [15] is formulated with the aim to adequately solve problems involving the factor of uncertainty and allowing the partial membership of an element to a certain set. The fuzzy theory uses a membership function to mathematically express the level of uncertainty. In this paper, triangular numbers are selected to show uncertainty when evaluating performances of simultaneous train formation methods (Figure 1):

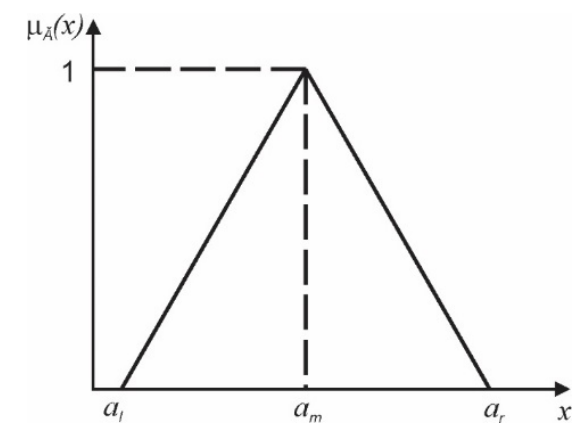

Fig. 1. Triangular fuzzy number Ã

$$
\mu_{A}(x)=\left\{\begin{array}{c}
0, x<a_{l} \\
\frac{x-a_{l}}{a_{m}-a_{l}}, \quad a_{l}<x<a_{m} \\
\frac{a_{r}-x}{a_{r}-a_{m},}, a_{m}<x<a_{r} \\
0, x>a_{r}
\end{array}\right\}
$$


A triangular fuzzy number can be defined as a triplet $\tilde{A}=\left(\alpha_{i}, \alpha_{m}, \alpha_{m}\right)$ where $a_{l}, a_{m}, a_{r}$ indicates, respectively, the lowest possible value, modal value and the highest possible value that describes a fuzzy number. The value $\mu_{A}(x)$ indicates the level of membership of an element x to the set $\tilde{A}$.

The operations on the triangular numbers $\tilde{A}=\left(a_{i}, a_{m}, a_{r}\right)$ and $\tilde{B}=\left(b_{l}, b_{m}, b_{r}\right)$ are defined by (2) - (7):

$$
\begin{gathered}
A+B=\left(a_{l}+b_{l}, a_{m}+b_{m}, a_{r}+b_{r}\right) \\
A-B=\left(a_{l}-b_{r}, a_{m}-b_{m}, a_{r}-b_{l}\right) \\
A \cdot \vec{B}=\left(a_{l} \cdot b_{l}, a_{m} \cdot b_{m}, a_{r} \cdot b_{r}\right) \\
k \cdot A=\left(k \cdot a_{l}, k \cdot a_{m}, k \cdot a_{r}\right) \\
\max (\tilde{A}, \vec{B})=\left(\max \left(a_{l}, b_{l}\right), \max \left(a_{m}, b_{m}\right), \max \left(a_{r}, b_{r}\right)\right) \\
\min (\widetilde{A}, \vec{B})=\left(\min \left(a_{l}, b_{l}\right), \min \left(a_{m}, b_{m}\right), \min \left(a_{r}, b_{r}\right)\right)
\end{gathered}
$$

The transformation of fuzzy numbers into appropriate classic numbers could be performed by centeroid method:

$$
x_{0}(\tilde{A})=\left(a_{l}+a_{m}+a_{r}\right) / 3
$$

\section{Proposed fuzzy multi-criteria approach}

Within the proposed model, the evaluation of selected simultaneous train formation methods $V_{j}(j=1,2, \ldots, m)$ according to the performance indicators $C_{i}(i=1,2, \ldots, n)$ is realized by the triangle fuzzy numbers $f_{i j}=\left(l_{i j}, m_{i j}, n_{i j}\right)$. The fuzzy VIKOR method algorithm consists of the following steps:

Step 1: Selection of the best $f_{i}^{*}=\left(l_{i}^{*}, m_{i}^{*}, r_{i}^{*}\right)$ and the worst $f_{i}^{-}=\left(l_{i}^{-}, m_{i}^{-}, r_{i}^{-}\right)$ values of the performance indicators:

$$
f_{i}^{*}=\min f_{i j}, f_{i}^{-}=\max _{j} f_{i j}
$$

Step 2: Determining a normalized value $\tilde{d}_{i j}, j=\mathbf{1}_{x}, \ldots, m, i=\mathbf{1}_{x}, \mathbf{2}_{,}, \ldots, n$ :

$$
d_{i j}=\frac{f_{i j}-f_{i}^{*}}{r_{i}^{-}-l_{i}^{*}}
$$

Step 3: Determining the value of the fuzzy distance measure $S_{j}=\left(S_{j}^{l}, S_{j}^{m}, S_{j}^{r}\right)$ and $\tilde{R}_{j}=\left(R_{j}^{L}, R_{j}^{m}, R_{j}^{r}\right)$ from the ideal solution: 


$$
\begin{aligned}
& \tilde{S}_{j}=\sum_{i=1}^{n}\left(\tilde{w}_{i} \cdot d_{i j}\right) \\
& \tilde{k}_{j}=\max _{i}\left(\tilde{w}_{i} \cdot \tilde{d}_{i j}\right)
\end{aligned}
$$

where $\widetilde{\boldsymbol{w}}_{i}$ is a fuzzy weight of the selected criteria.

Step 4: Determining the value of the fuzzy distance measure $\tilde{Q}_{j}, j=\mathbf{1}, 2, \ldots, m$ from the ideal point:

$$
\bar{Q}_{j}=\frac{\theta\left(s_{j}-s_{j}^{2}\right)}{s^{-r}-s^{2 !}}+\frac{(1-\theta)\left(A_{j}-A_{j}^{2}\right)}{R^{-T}-R^{2}}
$$

or

$$
\bar{Q}_{j}=\vartheta \widehat{Q S_{j}}+(1-\vartheta) \widetilde{Q R_{j}}
$$

where $S_{j}^{*}=\min _{j} \tilde{S}_{j}, S_{j}^{-}=\max _{j} S_{j}, \tilde{R}_{j}^{*}=\min _{j} \tilde{R}_{j}, \tilde{R}_{j}^{-}=\max _{j} \tilde{R}_{j}$, and $\vartheta$ is the weight of the decision-making strategy, $\vartheta=(0,1)$.

Step 5: Defuzzification of $\overrightarrow{Q S}_{j}, \overrightarrow{Q R}_{j}$ and $\widetilde{Q}_{j}$ values applying the relation (9).

Step 6: Ranking based on the defuzzified values $Q S_{j}, Q R_{j}$ and $Q_{j}$ and the proposal of a compromise solution.

In order to ensure that a variant method should be proposed as the most favourable one, it is necessary to meet the requirements of the "acceptable advantage" (condition C1) and "acceptable stability" (condition C2).

- Acceptable advantage (C1):

$$
Q\left(A^{r r}\right)-Q\left(A^{r}\right) \geq D Q
$$

where $A^{n}$ is the alternative with second position in the ranking list by $Q$ and $D Q=\min \{0.25,1 / m-1\}$, where $\mathrm{m}$ is the number of alternatives.

- Acceptable stability (C2):

- Alternative $A^{r}$ must also be the best ranked for $\vartheta=0.25$ and 0.75 , or either by $Q S$ and $Q R$.

\section{Numerical experiment}

In order to test our mathematical formulations we designed an illustrative example for selecting the most suitable train formation method among three simultaneous methods with fix sorting schedules: elementary $\left(\mathrm{V}_{1}\right)$, triangular $\left(\mathrm{V}_{2}\right)$ and geometric method $\left(\mathrm{V}_{3}\right)$. We defined four performance indicators: the number of wagon movements $\left(\mathrm{C}_{1}\right)$, the number of used tracks $\left(\mathrm{C}_{2}\right)$, total processing time $\left(\mathrm{C}_{3}\right)$ and complexity of shunting work $\left(\mathrm{C}_{4}\right)$.

We developed simulation models to enable modelling of sorting schedules based on [3, 7]. The following input parameters were adopted. We analyzed the case of sorting 150 wagons in order to compose 5 multi-group trains with about 30 wagons. The influence of the number of groups in the outgoing trains was tested by varying the number of groups from 4 to 15. Varying the number of groups we cover different problem levels: small, medium and large scale sorting tasks. The sorting task scales are represented as triplets: 
small (with 4, 5 and 6 groups), medium (with 8, 9 and 10 groups), and large (with 11, 13 and 15 groups). Such a wide range of groups for sorting tasks is assumed so that it can also be applicable to local or liner multigroup trains (the number of groups does not usually exceed 10), but also to industrial trains (the number of handling points can amount to 15 or more). For each case of the sorting task we generated 100 instances in order to obtain the indicators with sufficient level of relevance. The output results from simulation models are averaged and used to represent performance indicators $C_{1}$ to $C_{4}$ as triangular fuzzy numbers in the form $f_{i j}\left(l_{i j}, m_{i j}, r_{i j}\right)$. The triangular fuzzy numbers for the complexity of shunting work $\left(C_{5}\right)$ are obtained based on expert evaluations. All this values are presented in Table 1 as elements of the initial decision-making matrix.

Table 1. Initial decision-making matrix.

\begin{tabular}{|c|c|c|c|c|c|c|c|c|c|c|c|c|c|}
\hline & \multicolumn{3}{|c|}{$\begin{array}{c}\text { C1 } \\
\text { (No. } \\
\text { movements) }\end{array}$} & \multicolumn{3}{|c|}{$\begin{array}{c}\mathrm{C} 2 \\
\text { (No. tracks ) }\end{array}$} & \multicolumn{3}{|c|}{$\begin{array}{c}\text { C3 } \\
\text { (Min) }\end{array}$} & \multicolumn{3}{|c|}{$\begin{array}{c}\mathrm{C} 4 \\
\text { (Points) }\end{array}$} \\
\hline & & 1 & $\mathrm{~m}$ & $\mathrm{r}$ & 1 & $\mathrm{~m}$ & $\mathrm{r}$ & 1 & $\mathrm{~m}$ & $\mathrm{r}$ & 1 & $\mathrm{~m}$ & $\mathrm{r}$ \\
\hline \multirow{3}{*}{$\begin{array}{ll}\overline{\bar{\tau}} & \frac{0}{\tilde{J}} \\
\text { ज } & \end{array}$} & V1 & 150 & 150 & 150 & 4 & 5 & 6 & 196.4 & 224.5 & 249.6 & 1 & 1 & 10 \\
\hline & $\mathrm{V} 2$ & 194 & 200 & 226 & 5 & 6 & 7 & 242.7 & 286.6 & 337.1 & 1 & 5 & 10 \\
\hline & $\mathrm{V} 3$ & 194 & 200 & 226 & 5 & 6 & 7 & 242.7 & 286.6 & 337.1 & 1 & 5 & 10 \\
\hline \multirow{3}{*}{ 昙 $\frac{0}{\tilde{d}}$} & V1 & 150 & 150 & 150 & 8 & 9 & 10 & 262.5 & 281.6 & 300.7 & ]1 & 1 & 10 \\
\hline & $\mathrm{V} 2$ & 222 & 232 & 239 & 7 & 8 & 8 & 282.2 & 317.9 & 327.9 & 1 & 5 & 10 \\
\hline & V3 & 243 & 249 & 255 & 7 & 8 & 8 & 314.0 & 325.9 & 337.9 & 1 & 10 & 10 \\
\hline $\bar{\sigma} \quad$ & V1 & 150 & 150 & 150 & 11 & 13 & 15 & 358.7 & 399.4 & 439.5 & 1 & 1 & 10 \\
\hline
\end{tabular}

As performance indicators are expressed in different measurement units, it is required to normalize the initial matrix for decision-making (Step 1 and Step 2 of the fuzzy VIKOR method). For the realization of the ranking procedure, it is necessary to assign fuzzy weights coefficients $\tilde{w}_{i}$ to all the criteria and to determine fuzzy measures of distance $\tilde{S}_{j}$ and $\tilde{\boldsymbol{R}}_{j}$ of the variants from the ideal point (Step 3 ). Non-normalized values of weight coefficients are shown in Table 2 .

Table 2. Values of weight coefficients.

\begin{tabular}{|l|l|l|l|l|}
\hline & $\mathrm{C} 1$ & $\mathrm{C} 2$ & $\mathrm{C} 3$ & $\mathrm{C} 4$ \\
\hline$\tilde{w}_{i}\left(w_{i}^{l}, w_{i}^{m}, w_{i}^{\eta}\right)$ & $(1,1,1)$ & $(5,5,5)$ & $(3,3,3)$ & $(2,2,2)$ \\
\hline
\end{tabular}

Based on the adopted weight of the decision-making strategy $\theta=0.5$, fuzzy measures of distance $\phi_{j}$ have been determined (Step 4). After the defuzzification of the values $\overline{Q S}, \overline{Q R}$ and $\bar{C}$ (Step 5) the final decision-making matrix and ranking lists are made for following measures of distance QS, QR, and Q, as shown in the Table 3 (Step 6). 
Table 3. Ranking lists.

\begin{tabular}{|c|c|c|c|c|c|c|c|}
\hline \multirow{3}{*}{\multicolumn{2}{|c|}{ Scenarios }} & \multicolumn{6}{|c|}{ Variant solution } \\
\hline & & \multicolumn{2}{|l|}{ V1 } & \multicolumn{2}{|l|}{ V2 } & \multicolumn{2}{|l|}{ V3 } \\
\hline & & Value & Rank & Value & Rank & Value & Rank \\
\hline \multirow{3}{*}{ Small scale } & Qs & 0,000 & 1 & 0,241 & 2 & 0,241 & 3 \\
\hline & $Q R$ & 0,000 & 1 & 0,279 & 2 & 0,79 & 3 \\
\hline & $Q$ & 0,000 & 1 & 0,260 & 2 & 0,260 & 3 \\
\hline \multirow{3}{*}{ Medium scale } & QS & 0,033 & 2 & 0,059 & 1 & 0,165 & 3 \\
\hline & $Q R$ & 0,142 & 3 & 0,034 & 1 & 0,120 & 2 \\
\hline & $Q$ & 088 & 2 & 0,047 & 1 & 0,143 & 3 \\
\hline \multirow{3}{*}{ Largel scale } & QS & 0,169 & 3 & 0,000 & 1 & 0,165 & 2 \\
\hline & $Q R$ & 0,435 & 3 & 0,000 & 1 & 0,192 & 2 \\
\hline & $Q$ & 0,302 & 3 & 0,000 & 1 & 0,179 & 2 \\
\hline
\end{tabular}

The results of the multi-criteria evaluation show that elementary method $\left(\mathrm{V}_{1}\right)$ is the first in the ranking for small scale sorting task. Even more, the elementary method can be chosen as the most favourable sorting schedule as both requirements regarding the acceptable advantage and stability are fulfilled. In the case of simultaneous train formation with small number of groups, the elementary method has advantages as it does not require high number of tracks, as well as it realizes sorting procedure with the minimum volume of shunting work. Note that, the number of movements is equal for all groups and amounts to precisely one movement per wagon. In the case of medium scale sorting task, triangular method $\left(\mathrm{V}_{2}\right)$ is the first ranked followed by elementary method $\left(\mathrm{V}_{1}\right)$ and geometric method $\left(\mathrm{V}_{3}\right)$. Although triangular method is first ranked, it can't be directly chosen as the most favourable method for medium scale sorting tasks, as it does not fulfilled requirement regarding the acceptable advantage. It means that for each specific medium scale sorting task is required to analyse performances of all methods in order to make adequate decision. In the case of large scale sorting tasks, triangular method is also first ranked fulfilling the requirement regarding the acceptable stability. Even more, it has sufficient advantage over elementary method, but not over geometric method. It means that for large scale sorting tasks, we can exclude elementary method as it requires high number of sorting tracks. However, we need to make additional considerations regarding the application of geometric method as it provides more complex sorting schedule which can in some situations to drastically reduce the number of required sorting tracks.

\section{Conclusions}

Economy of scale benefits are main advantages of wagonload transportation that keep the interest among shippers in such services. These facts confirm the potential of wagonload transportation. In order to be more competitive to road transport in this transportation area, railways need to improve speed, reliability and cost effectiveness of wagonload services. Multi-group trains have potential to take a substantial segment in realizing wagonload services. Multi-group trains are dominantly used as industry trains or for "last mile" service as local freight trains but their use in long-distance transport is viable also.

The application of multi-group train service leads to the reduction of total layover time of wagons and to the concentration of shunting work on smaller number of main marshalling yards. However, the complex marshalling procedure regarding multi-group train formation influences the performance of wagonload services. In that sense, the proper 
application of simultaneous train formation methods can increase the efficiency of the wagonload system.

The approaches used in the existing literature in evaluating train formation methods are mainly based on the mathematical optimization expressed through the minimization of wagon movements or pull-out operations. However, the determination of rational sorting schedules should be based on more comprehensive approach considering different technical and technological aspects. These aspects commonly address quantitative and qualitative indicators which are conflicting and uncertain. In order to address these conditions, we proposed a new approach for fuzzy multi-criteria evaluation of simultaneous train formation methods. In this paper we evaluated simultaneous methods with elementary, triangular and geometric sorting schedules. Conducted numerical experimentations proven that the elementary sorting is the favorable method for simultaneous formation of trains composed of smaller number of groups. Increasing the number of groups, triangular and geometric sorting are getting more suitable. Finally, in the case of higher number of groups, triangular and geometric sorting prevail over elementary sorting as they require considerable smaller number of sorting tracks.

Acknowledgements. This paper is realized and supported by The Ministry of Educations and Science of the Republic of Serbia, in the frame of the project: "Research of technical-technological, staff and organizational capacity of Serbian Railways, from the viewpoint of current and future European Union requirements", evidential number TR 36012.

\section{References}

1. M. Vilotijević, M. Vukićević, et al., Technical Gazette 25, 2 (2018).

2. Z. Popović, L. Lazarević et al., MATEC Web of Conferences 106, 05001 (2017).

3. M. Ivić, I. Belošević et al., Građevinar 65, 123-134 (2013).

4. I. Belošević, M. Ivić et al., 2nd Logistics International Conference, 62-67 (2015).

5. I. Belošević, M. Ivić et al., Horizons Series 3, 559-569 (2016).

6. P. Márton, Communications 2, 5-8 (2005).

7. I. Belošević, M. Ivić et al., Građevinar 64, 553-563 (2012).

8. R. Jacob, P. Márton et al., Networks 57, 87-105 (2011).

9. I. Belošević, M. Ivić: Computer-Aided Civil and Infrastructure Engineering 33, 220242 (2018).

10. M. Gul, E. Celik et al., Applied Soft Computing 46, 60-89 (2016).

11. I. Belošević, M. Kosijer et al., European Transport Research Review 10, 43 (2018).

12. I. Belošević, S. Milinković et al., MATEC Web of Conferences 235, 00019 (2018).

13. P. Márton, S. Milinković et al., Transportation Research Procedia 40, 1459-1464 (2019).

14. S. Opricovic, G.H. Tzeng, European Journal of Operational Research 178, 514-529 (2007).

15. L.A. Zadeh Information and Control 8, 338-353 (1965). 